\title{
USING MULTI-WAVELENGTH ALL SKY INFORMATION TO UNDERSTAND LARGE SCALE GALACTIC STRUCTURE.
}

\author{
R.L. SMART, R. DRIMMEL AND M.G. LATTANZI \\ Osservatorio Astronomico di Torino
}

\section{Introduction}

Our galaxy was first seen to be warped, similarly to other galaxies, in $21 \mathrm{~cm}$ HI surveys (Kerr 1957). Since then a large database on our and other warps has been built but they remain a puzzle for theorists trying to construct a general warp theory. Warps are observed to be common rather than rare features of spiral galaxies, which implies they are long lived. They are observed in both isolated and multiple galaxy systems in the gas but the stellar warp is often difficult to detect. The galactic warp is always below $\mathrm{b}=10^{\circ}$ making it difficult to observe because of absorption and confusion, to add to this problem the sun appears to lie near to the line of nodes and to view the warp at it's largest extent we must observe through a large part of the disk. A theoretical model must evoke driving forces that explain the longevity, commonality, and independence of environment that we see in warps.

\section{Previous Work}

Observations of the HI $21 \mathrm{~cm}$ line indicate the southern half of the galactic warp curving back towards the plane after reaching an amplitude of 1.5 $\mathrm{kpc}$ at radius $16 \mathrm{kpc}$, while the northern half continues to rise, reaching $4 \mathrm{kpc}$ at $23 \mathrm{kpc}$ (Burton 1991). Wouterlott et al. (1990) analyzed CO molecular cloud observations and showed these are consistent with the HI observations.

The stellar warp is difficult to observe for many reasons: absorption in the plane, confusion of warp stars with foreground or background stars, an apparent (related?) cutoff in the stellar density near the radius the warp 
begins, and a possible non-coincidence of the gas and stellar warp makes it difficult to predict where the stellar warp is. Early work by Guibert et al. (1978) showed that the positions of young star populations are consistent to a plane model both with and without a warp. More recent work on OB stars (Cameron-Reed 1996 and references therein) and Cepheids (Efremov et al. 1981) implies the young star plane is warped. This maybe confused because of their proximity to their birthplace in the HI plane.

Carney \& Seitzer (1993) show an overabundance of stars in the HI warped direction compared to fields at the same longitude but opposite latitude. Djorgovski \& Sosin (1989) examined AGB stars in the IRAS Point Source Catalog showing a warped plane. These both indicate that the old stellar disk is also warped. Freudenreich et al. (1994) looked at the results of the Diffuse Infrared Background Experiment on COBE to map out the surface brightness of the dust plane in our galaxy and found it was consistent with the $\mathrm{HI}$ observations but indicated different parameters. All of these three studies examine the projected warp, e.g., there is very little distance information and, therefore, room for other interpretations.

Therefore, for our galaxy, on a first examination observations indicate a consistent warped plane for all the constituents, young and old stars, dust and gas with that caveat that there is room for different warp parameters of the constituents. Observations of our galaxy and other galaxies indicate these parameters are different. Freudenreich et al. (1994) mentioned above found different parameters for the gas and dust. Miyamoto et al. (1994), looking at proper motions of old K/M giants and young O/B stars, found that the plane parameters differed significantly, with the young stars following the HI plane and the old stellar plane unwarped. Florido et al. (1991) observed three galaxies in four colors, basically sampling different constituents, and found that the warp had a significant variation. Finally on a more fundamental note, if all the constituents follow the same warped plane it strongly implies that the driving force is gravity as other possibilities, e.g., intergalactic winds or magnetic fields, would effect dust and gas quite differently than the stars. However, Battaner et al. (1991) found a relationship between warp orientations of nearby galaxies which is difficult (if not impossible) to explain with warps originating from gravitational fields.

\section{Problems and Future Work}

One of the main problems here is with the differing modes of interpretation. With HI and CO data distances are found using a rotation curve and gas velocities, for the $\mathrm{OB}$ and cepheids usual photometric distance techniques are employed and for the old stars and dust observations minimal distance 

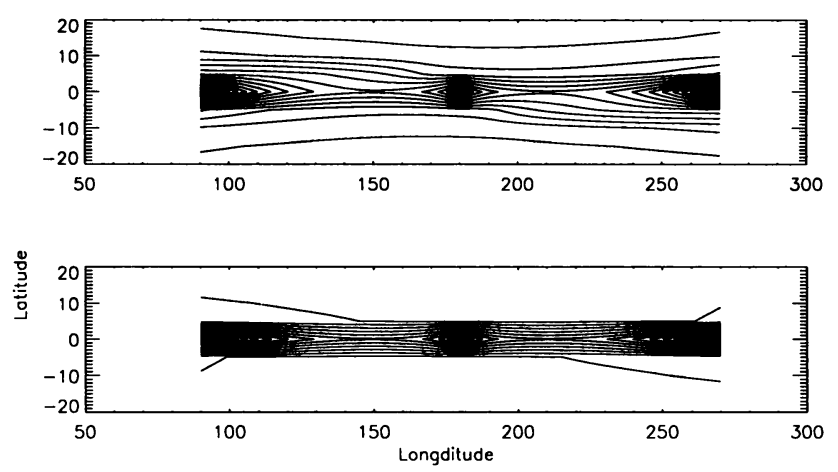

Figure 1. Contour plots of stellar number counts in a warped plane without and with absorption. When absorption is added the warp effect in number counts disappears.

information is known. An example of the many problems here is shown in Figure 1, which compares the number counts of a warped stellar plane with and without absorption from a simple galactic model. Similar examples can be made for other systematic errors introduced by, for example, noncircular rotation curves, miscalibration of photometric distance estimates, etc.

At Torino we have undertaken two projects to examine the warp of our galaxy. One is to interpret the integrated observations of the dust and old stars using three dimensional models. The second is to compare the HIPPARCOS observations of O/B stars to simulated galactic models of these stars. Using HIPPARCOS results has a number of advantages, they are all sky and complete to a well understood level, the kinematical information provides more constraints than just spatial data alone, they are very precise and free from systematic zonal effects that hindered the Miyamoto et al. work. Finally, TYCHO, the HIPPARCOS star mapper, will provide precise colors on a consistent system for finding accurate photometric parallaxes.

\section{Acknowledgements}

RLS thanks the SOC for support. to attend this meeting. We thank Stefano Casertano, James Binney and David Spergel for useful comments.

\section{References}

Battaner E., Floridao E. \& Sanchez-Saavedra M.L. 1990 Astron.Astrophys. 236, 1. Binney J. 1992, Ann.Rev.Astron.Astrophys. 30, 51

Burton W.B. 1991 SAAS-FEE 21, eds. W.B. Burton, B.G. Elmgreen \& R. Genzel.

Cameron Reed B. 1996 Astron.J. 111,804.

Carney B.W. \& Seitzer P. 1993 Astron.J. 105, 2127.

Djorgovski S. \& Sosin C. 1989 Astrophys.J. 341, L13. 
Efremov Y.N., Ivanov G.R. \& Nikolov N.S., 1981 Astron.Sp.Sc 75, 407.

Freudenreich H.T., Berriman G.B., Dwek, E., Hauser, M.G., Kelsall T., Moseley S.H., Silverberg R.F., Sodroski T.J., Toller G.N., \& Weiland J.L. 1994 Astrophys.J.Lett. 429, L69.

Florido, E., Battaner, E., Sanchez-saavedra, M. L.,Prieto M. \& Mediavilla, E. 1991 Astron.Astrophys. 242, 301.

Guibert J., Lequeux J. \& Viallefond F. 1978 Astron.Astrophys. 68, 1

Kerr F.J. 1957 Astron.J. 62, 93.

Miyamoto M., Yoshizawa M., \& Suzuki S. Astron.Astrophys. 194, 107.

Wouterloot J.G.A., Brand J., Burton W.B., \& Kwee K.K. 1990 Astron.Astrophys. 230, 21. 